\title{
Matrix effects on the optical response of silver nanoclusters
}

\author{
F. Conus, V. Rodrigues, ${ }^{\text {a) }}$ S. Lecoultre, A. Rydlo, and C. Félix ${ }^{\text {b) }}$ \\ Institut de Physique des Nanostructures, EPFL, CH-1015 Lausanne, Switzerland
}

(Received 4 May 2006; accepted 9 June 2006; published online 14 July 2006)

\begin{abstract}
We report absorption spectra for $\mathrm{Ag}_{7}, \mathrm{Ag}_{9}$, and $\mathrm{Ag}_{11}$ in an argon matrix grown at a temperature of $28 \mathrm{~K}$ and compare them with previous spectra of the same species measured in matrices of argon grown at lower temperatures as well as in neon matrices. We discuss the discrepancies in the light of the matrix crystallinity and show that this leads to an understanding of the influence of the matrix on the optical response of small clusters. (C) 2006 American Institute of Physics.
\end{abstract}

[DOI: 10.1063/1.2219442]

\section{INTRODUCTION}

Nanoclusters have interesting and promising properties in domains as diverse as optics, ${ }^{1-4}$ catalysis, ${ }^{5,6}$ and magnetism. ${ }^{7-10}$ While some of these properties can arise for the cluster itself and can be studied in the gas phase, in most practical cases the nanoclusters are in interaction with their environment. This interaction is often not well understood but can have important effects on the physical properties. We propose here an experimental study of this interaction on a model system: silver clusters in a rare gas matrix that should be relatively inert. It demonstrates that even in such an "ideal" system the detailed local environments of the clusters have strong effects on their optical response.

Optical spectroscopy of small mass selected metal clusters is not an easy task for the absorption cross sections are relatively small. Such systems have been mainly studied either by photodepletion spectroscopy on cluster-rare gas complexes $^{11}$ or in an embedding rare gas matrix, ${ }^{12}$ where they are accumulated to build up a sufficient integrated density for the absorption signal to be measurable.

Even though rare gas matrices are relatively inert, the effect of the matrix on the optical spectrum remains an open question. Fedrigo et al. ${ }^{13}$ have discussed the role of the embedding matrix dielectric constants on the absorbtion spectra for different rare gases. Later, Andersen and Bonderup ${ }^{14}$ corrected his simple description by considering the local field corrections for light absorption using the Onsager cavity model.

In addition to the purely dielectric effect, there can be different embedding sites depending on the exact location of the neighboring atoms, resulting in different absorption spectra. In a crystallized matrix, clusters with differing but well defined local environments, known as site isomers, may coexist. This has been observed, for example, in $\mathrm{Ag}_{2}$ and $\mathrm{Ag}_{3} \cdot{ }^{15-17}$ Using quantum calculations on sodium clusters embedded in an argon matrix, Gervais et al. ${ }^{18,19}$ showed the possible coexistence of several site isomers with slightly different transition energies and intensities. The effect of the

\footnotetext{
a) Present address: Instituto de Física "Gleb Wataghin," UNICAMP, C.P 6165, 13083-970 Campinas SP, Brazil.

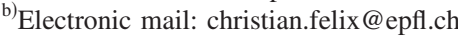

matrix is shown to be the result of two competing effects: the dielectric effect discussed above that leads to a redshift and a confinement of the valence electrons of the cluster leading to a blueshift.

In this study we present absorption spectra for $\mathrm{Ag}_{7}, \mathrm{Ag}_{9}$, and $\mathrm{Ag}_{11}$ in argon acquired with a new setup. ${ }^{20}$ To enhance matrix transparency, our matrix was grown at a higher temperature $(28 \mathrm{~K})$ compared to previous measurements $(10 \mathrm{~K}),{ }^{12}$ showing unexpected differences. We discuss these discrepancies in the light of the different matrix growth conditions and show that this leads to an understanding of the influence of the matrix on the optical response of small clusters.

\section{EXPERIMENTAL SETUP}

The experimental setup has been described in detail elsewhere. ${ }^{20}$ Briefly, clusters are formed from a metal target sputtered by a $15 \mathrm{~mA} \mathrm{Xe}{ }^{+}$ion current at $25 \mathrm{keV}$. The cluster cations are guided to a quadrupole mass filter and then to a cold sample holder $(28 \mathrm{~K})$, where they are deposited together with argon and neutralized to form a seeded rare gas matrix of neutral species. The clusters are accumulated in the matrix for $2.5 \mathrm{~h}$ while a $40 \mu \mathrm{m}$ thick matrix is grown. A capping layer of argon is then grown on top of the matrix to protect it from any contamination and to optimize the optical transmission of the matrix.

Optical absorption is then performed through the $2 \mathrm{~mm}$ length of matrix. ${ }^{20}$ White light is injected into the matrix and collected on the other side with an optical fiber. This geometry allows an increase in sensitivity compared to previous measurements ${ }^{12}$ due to the longer optical path. The collected light is analyzed by an optical spectrometer coupled to a liquid-nitrogen-cooled charge coupled device (CCD). Comparing the signal of light passing through a matrix doped with clusters with a reference signal of light passing through a pure argon matrix yields the absorption spectrum of the deposited species.

\section{RESULTS}

Figure 1 compares the absorption spectra of $\mathrm{Ag}_{7}$ in a matrix grown at $28 \mathrm{~K}$ (this work), in an argon matrix grown at $10 \mathrm{~K},{ }^{21}$ and in a neon matrix grown at $5 \mathrm{~K}^{22}$ There is a 


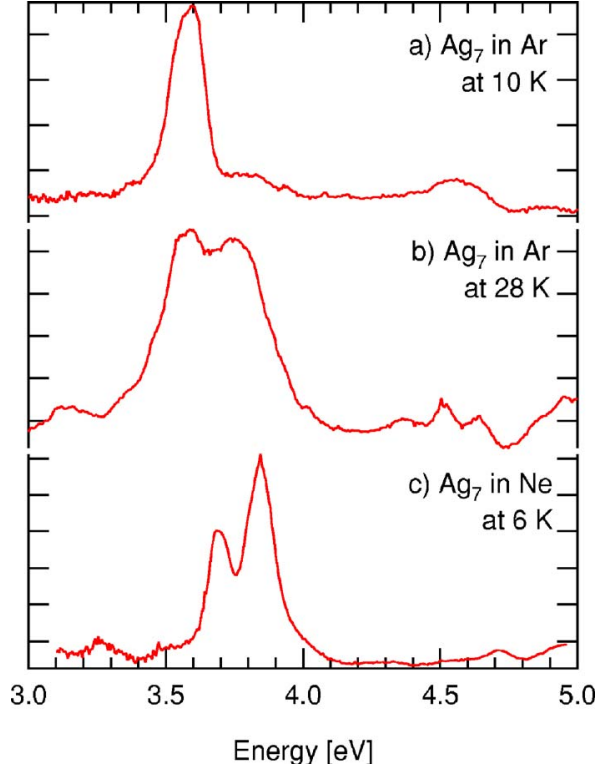

FIG. 1. Absorption spectra of $\mathrm{Ag}_{7}$. (a) In an argon matrix grown at $10 \mathrm{~K}$ (Ref. 21). (b) In an argon matrix grown at $28 \mathrm{~K}$ (this work). (c) In a neon matrix at $6 \mathrm{~K}$ (Ref. 22).

remarkable difference in the absorption spectra recorded in argon matrix depending on the growth temperature. While the spectrum in the matrix grown at $10 \mathrm{~K}$ shows essentially a single peak $(3.58 \mathrm{eV})$, one observes the appearance of a second peak $(3.75 \mathrm{eV})$ in the "warm" matrix spectrum. A shoulder in the $10 \mathrm{~K}$ spectrum is an onset of this peak. The second peak is also present in a neon matrix, with, however, a slight shift towards the blue by $0.1 \mathrm{eV}$ due to different dielectric constants of the matrices. ${ }^{13}$ Less intense features are also visible at $3.22 \mathrm{eV}$, both in the warm argon and the neon matrices. At higher energies, three peaks are distinguishable at $4.37,4.51$, and $4.64 \mathrm{eV}$ while they are blurred out in a single broad feature in the argon matrix at $10 \mathrm{~K}$. For the neon, one can guess a single feature of very low intensity. While the positions of the peaks show a very good agreement in Figs. 1(b) and 1(c), given the $0.1 \mathrm{eV}$ shift, one notices that the relative intensities between the two main peaks is reversed.

A similar trend can be observed for $\mathrm{Ag}_{9}$, on Fig. 2. We

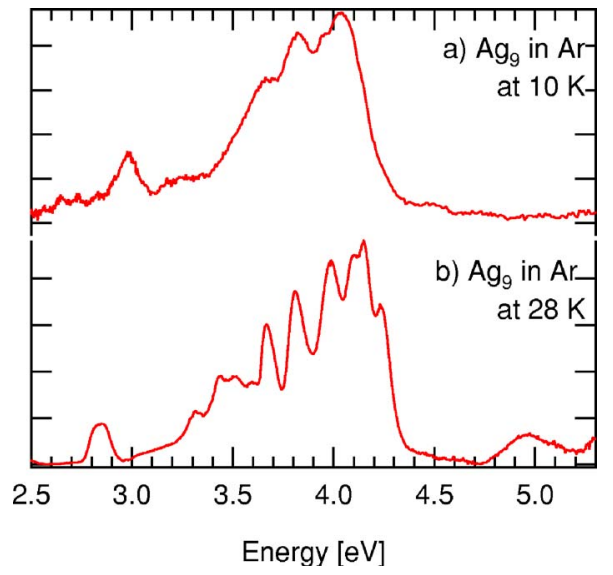

FIG. 2. Absorption spectra of $\mathrm{Ag}_{9}$ in an argon matrix. (a) Matrix grown at $10 \mathrm{~K}$ (Ref. 21). (b) matrix grown at $28 \mathrm{~K}$ (this work).

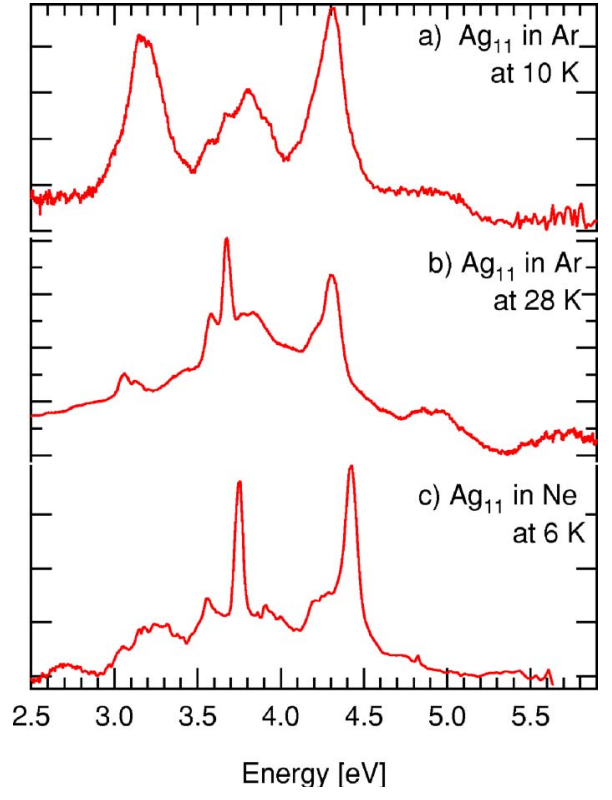

FIG. 3. Absorption spectra of $\mathrm{Ag}_{11}$. (a) In an argon matrix grown at $10 \mathrm{~K}$ (Ref. 21). (b) In an argon matrix grown at $28 \mathrm{~K}$ (this work). (c) In a neon matrix (Ref. 23).

compare again an absorption spectrum for clusters deposited in a matrix grown at $28 \mathrm{~K}$ to the same system but with a matrix grown at $10 \mathrm{~K}^{21}$ No absorption spectrum in neon is available for $\mathrm{Ag}_{9}$. The detailed features are narrower and the number of transitions individually addressable is more abundant in the matrix grown at $28 \mathrm{~K}$ compared to the one grown at $10 \mathrm{~K}$; we notice, however, that the overall spectrum is larger in energy. The new peak positions are at 2.84, 3.32, $3.44,3.51,3.59,3.67,3.81,3.99,4.10,4.15$, and $4.23 \mathrm{eV}$ and a broader peak centered at $4.95 \mathrm{eV}$. The width of the peak at $2.84 \mathrm{eV}[\sim 110 \mathrm{meV}$ full width at half maximum (FWHM)] is higher than the width of the other peaks $\sim 70 \mathrm{meV}$ and may be due to two different transitions. It is also redshifted by $0.14 \mathrm{eV}$ compared to the corresponding peak at $10 \mathrm{~K}$.

Figure 3 shows the absorption spectra of $\mathrm{Ag}_{11}$ obtained in argon matrices grown at 28 and $10 \mathrm{~K}$ (Ref. 21) as well as in neon matrix. ${ }^{23}$ For argon, the overall positions of the three main transitions $(3.06,3.67$, and $4.31 \mathrm{eV})$ are in close agreement, however, the peaks are broader in the spectrum obtained at lower temperature and the relative intensities are different. Some weaker transitions are observed only for the matrix grown at $28 \mathrm{~K}$, they are situated at 3.12, 3.41, 3.58, $3.83,4.22,4.85$, and $4.98 \mathrm{eV}$. As for $\mathrm{Ag}_{7}$, the similarity between the spectra recorded in argon matrix grown at $28 \mathrm{~K}$ and in neon matrix grown at $6 \mathrm{~K}$ is striking, being the peaks shifted by $0.1 \mathrm{eV}$ to the blue due to the dielectric properties of the medium.

\section{DISCUSSION}

As mentioned above, we are recording the absorption spectra through the width of the matrix rather than through its thickness in order to increase the sensitivity. The optical path is three orders of magnitude longer than in the work by Harbich et al. ${ }^{21}$ To circumvent the severe scattering of the 
argon matrix in the UV, the matrices need to be grown at higher temperature $(T=28 \mathrm{~K}$ instead of $10 \mathrm{~K}$ for Harbich et al.) and have therefore an improved crystallinity. We do not have to fear interferences in the matrix since the optical path is long compared to the used wavelengths.

The present data show that the increase in the growth temperature does not only change the transmission of the matrix but also the spectral features of the embedded clusters. To exclude experimental artifacts, we have verified that $\mathrm{Ag}_{1}$ (Ref. 20) showed exactly the same spectrum with both setups. Also, the strong similitudes between the spectra recorded in the warm argon matrix and neon matrix for $\mathrm{Ag}_{7}$ and $\mathrm{Ag}_{11}$ suggest that it is not an artifact. We therefore attribute the origin of the differences to the growing temperatures that modifiy the crystallinity of the matrix.

According to Fugol', ${ }^{24}$ one can distinguish between different rare gas matrix growth regimes. When the condensation temperature is above $2 / 3$ of the sublimation temperature of the rare gas, the matrix grows as nanocrystallites of more than $100 \mathrm{~nm}$. Below or equal to $1 / 3$ of the sublimation temperature it grows as nanocrystallites of less than $10 \mathrm{~nm}$ and shows lots of defects. The sublimation temperature of argon being approximately $30 \mathrm{~K}$, one sees that the matrix grown at $10 \mathrm{~K}$ enters the second category, while ours is in the first one. This suggest that the local order of the argon atoms around a given cluster is better defined for the warm matrices.

This conclusion is corroborated by the fact that neon matrices are known for their higher crystallinity and that the absorption spectra of $\mathrm{Ag}_{11}$ in a neon matrix [Fig. 3(c)] and in argon grown at $28 \mathrm{~K}$ [Fig. 3(b)] are very similar. This also indicates that the local order of the matrix has a more crucial importance on the measured spectrum than the detail of the local forces that are exerted by the neighboring rare gas atoms of the cluster.

It is interesting to notice that the transition from broad plasmonlike features to narrower molecularlike features, when the matrix deposition temperature increases, resembles what has been observed for sodium cation clusters in the gas phase. ${ }^{25}$ At low temperature, molecular spectra with well defined and narrow peaks were observed. When increasing the temperature, the spectra were less resolved, some peaks merged, and could be seen as plasmonlike features. This transition was interpreted as the transition from a molecule at low temperature to a liquid cluster at higher temperatures. The signal at higher temperature has been discussed as an absorption signal averaged on an ensemble of structures leading to an inhomogeneous broadening of the transition peaks. $^{26}$

We suggest that something similar happens here. For the $10 \mathrm{~K}$ case, absorption signal is averaged on an ensemble of structures that are in different embedding sites that coexist for matrices grown at low temperature. For matrices at higher temperature, some or most of these sites could be annealed, leading to similar cluster environs.

On the theoretical part, recent work by Gervais et al. ${ }^{18}$ has shown that the surrounding matrix modifies slightly the geometry of the embedded cluster and therefore its electronic structure, changing its optical response. Moreover the pres- ence of the matrix produces a confinement of the valence electrons of the cluster resulting in a blueshift of the transitions. If the local order around a cluster in a given trapping site is not well defined, one can easily imagine that the orientation of the cluster can slightly vary around the ideal position, thus modifying its optical response and leading to an inhomogeneous broadening of the transitions. The quality of the matrix therefore explains the different relative intensities observed when comparing the two spectra obtained in argon.

For $\mathrm{Ag}_{7}$ the spectrum is sufficiently simple that we can reasonably think of a single geometrical structure in the matrix. This is corroborated by calculations ${ }^{27-29}$ that indicate the pentagonal bipyramid structure as the lowest energy isomer, separated by a few hundreds of meV from the second lowest isomer, that has a tricapped tetrahedron arrangement. The simulated absorption spectrum by Bonačić-Koutecký et al. ${ }^{28}$ shows that a single dominant transition is expected for the lowest energy isomer. This is in good agreement with the experimental spectrum in argon matrix grown at $10 \mathrm{~K}$, taking into account a shift of $0.25 \mathrm{eV}$ due to the dieletric effect of the matrix. This agreement is surprising since we would have expected that the simulated spectrum resembles the warm argon matrix and neon data. These spectra show clearly two dominant transitions. How can this discrepancy be understood? We believe that it does not necessarily invalidate our previous analysis for different reasons. The presence of a neighboring atom from the matrix or simply attached to $\mathrm{Ag}_{7}$ could allow a transition that is otherwise symmetry forbidden, thus explaining the presence of a second peak in the spectrum. Alternatively this second peak could result from a deformation of the cluster by the force exerted by the matrix; since measured photodepletion spectrum ${ }^{11}$ also shows two peaks, we favor the first explanation. Finally we notice that other calculated spectra by Idrobo et al. ${ }^{29}$ predict one dominant transition as well as several other transitions with appreciable oscillator strength in the visible.

In the case of $\mathrm{Ag}_{9}$ we showed in a previous publication ${ }^{3}$ that the absorption signal for the matrix grown at $10 \mathrm{~K}$ is due to the coexistence of several isomers in the matrix. The large number of narrow peaks observed in argon deposited at $28 \mathrm{~K}$ [Fig. 2(b)] confirms our previous conclusions. The calculated spectra for the lowest energy isomers ${ }^{3}$ all have simpler spectral signatures than the measured spectrum, confirming the coexistence of different low energy isomers in the matrix.

Interestingly, the absorption spectrum in a warm matrix has sharper peaks that are distributed on a wider energy region than the absorption peaks of a "cold" matrix. The inhomogeneous broadening of the peaks is accompanied by a concentration of the oscillator strength on the dominant peaks. The same phenomenon is visible in the spectra of Ellert et al. ${ }^{25}$

$\mathrm{Ag}_{11}$ shows a high number of narrow features in the absorptions spectrum. As for $\mathrm{Ag}_{7}$ the very strong similitude between the spectra recorded in neon and in argon grown at $28 \mathrm{~K}$ indicates that the shifts due to the deformation of the clusters by the presence of the matrix are probably weak. Here we favor an explanation due to the coexistence of low energy isomers. The calculations by Fournier $^{27}$ show that there are several isomers relatively close in ground state en- 
ergy, the exact energy differences varying somewhat with the exact method used. It would be interesting to perform excitation spectroscopy on this system to confirm this hypothesis.

\section{CONCLUSION}

In this work we have made a systematic comparison between the absorption spectra of silver clusters in an argon matrix, obtained with our new experimental setup and previously recorded spectra in argon and in neon matrices. The spectra obtained for $\mathrm{Ag}_{11}$ and $\mathrm{Ag}_{9}$ in argon matrices condensed at $10 \mathrm{~K}$ show broader features than for argon matrices grown at $28 \mathrm{~K}$ or for neon matrices. This can be explained by considering a better defined local order of the rare gas atoms around the clusters in the case of the matrices grown at $28 \mathrm{~K}$ and the neon matrices. In the case of $\mathrm{Ag}_{7}$, this change in the local order leads to a splitting of the unique peak measured in the $10 \mathrm{~K}$ argon matrix into two distinct transitions that are observed in the $28 \mathrm{~K}$ matrix as well as in the neon matrix. This study is an experimental demonstration that an "inert" matrix can have a dramatic effect on the optical response of an embedded cluster of few metal atoms. The local order of the matrix atoms around the cluster is a crucial parameter that can change the optical response of the chromophore, due to a change of its geometry and/or electronic structure. This is a clear experimental demonstration that the detailed optical properties of nanoclusters depend on substrate effects, even if the substrate is almost inert; thus validating the route followed by Gervais et al.

${ }^{1}$ C. Félix, C. Sieber, W. Harbich, J. Buttet, I. Rabin, W. Schulze, and G. Ertl, Phys. Rev. Lett. 86, 2992 (2001).

${ }^{2}$ L. A. Peyser, A. E. Vinson, A. P. Bartko, and R. M. Dickson, Science 291, 103 (2001).

${ }^{3}$ C. Sieber, W. Harbich, J. Buttet, C. Félix, R. Mitrić, and V. BonačićKoutecký, Phys. Rev. A 70, 041201 (2004).
${ }^{4}$ T. H. Lee, J. I. Gonzales, and R. M. Dickson, Proc. Natl. Acad. Sci. U.S.A. 99, 10272 (2002)

${ }^{5}$ A. Sanchez, S. Abbet, U. Heiz, W.-D. Schneider, R. N. B. H. Häkkinen, and U. Landman, J. Phys. Chem. 103, 9573 (1999).

${ }^{6}$ B. Yoon, H. Hakkinen, U. Landman, A. S. Worz, J.-M. Antonietti, S. Abbet, K. Judai, and U. Heiz, Science 307, 403 (2005).

${ }^{7}$ I. M. L. Billas, A. Chatelain, and W. A. de Heer, Science 265, 1682 (1994).

${ }^{8}$ J. T. Lau, A. Fohlisch, R. Nietubyc, M. Reif, and W. Wurth, Phys. Rev. Lett. 89, 057201 (2002).

${ }^{9}$ P. Gambardella, S. Rusponi, M. Veronese et al., Science 300, 1130 (2003).

${ }^{10}$ S. Serrano-Guisan, G. di Domenicantonio, M. Abid, J.-P. Abid, M. Hillenkamp, L. Gravier, C. F'elix, and J.-P. Ansermet, Nature Materials (in press).

${ }^{11}$ D. Rayner, K. Athanassenas, B. Collings, S. Mitchell, and P. Hackett, in Theory of Atomic and Molecular Clusters: With a Glimpse at Experiments, edited by J. Jellinek (Springer, Berlin, 1999), p. 371.

${ }^{12}$ S. Fedrigo, W. Harbich, and J. Buttet, Phys. Rev. B 47, 10706 (1993).

${ }^{13}$ S. Fedrigo, W. Harbich, and J. Buttet, Int. J. Mod. Phys. B 6, 23 (1992).

${ }^{14}$ J. Andersen and E. Bonderup, Eur. Phys. J. D 11, 435 (2000).

${ }^{15}$ P. S. Bechthold, U. Kettler, and W. Krasser, Surf. Sci. 156, 875 (1985).

${ }^{16}$ U. Kettler, P. S. Bechthold, and W. Krasser, Surf. Sci. 156, 867 (1985).

${ }^{17}$ I. Rabin, W. Schulze, G. Ertl, C. Félix, C. Sieber, W. Harbich, and J. Buttet, Chem. Phys. Lett. 320, 59 (2000).

${ }^{18}$ B. Gervais, E. Giglio, E. Jacquet, A. Ipatov, P.-G. Reinhard, F. Fehrer, and E. Suraud, J. Chem. Phys. 121, 8466 (2004).

${ }^{19}$ B. Gervais, E. Giglio, E. Jacquet, A. Ipatov, P.-G. Reinhard, F. Fehrer, and E. Suraud, Phys. Rev. A 71, 015201 (2005).

${ }^{20}$ F. Conus, J. T. Lau, V. Rodrigues, and C. Félix (unpublished).

${ }^{21}$ W. Harbich, S. Fedrigo, and J. Buttet, Chem. Phys. Lett. 195, 5 (1992).

${ }^{22}$ S. Fedrigo, W. Harbich, and J. Buttet (unpublished).

${ }^{23}$ W. Harbich, Y. Belyaev, R. Kleiber, and J. Buttet, Surf. Rev. Lett. 3, 1147 (1996)

${ }^{24}$ I. Y. Fugol', Adv. Phys. 27, 1 (1978).

${ }^{25}$ C. Ellert, M. Schmidt, C. Schmitt, T. Reiners, and H. Haberland, Phys. Rev. Lett. 75, 1731 (1995)

${ }^{26}$ V. Bonačić-Koutecký, in Metal Clusters, Wiley Series in Theoretical Chemistry, edited by W. Ekardt (Wiley, Chichester, 1999).

${ }^{27}$ R. Fournier, J. Chem. Phys. 115, 2165 (2001).

${ }^{28}$ V. Bonačić-Koutecký, V. Veyret, and R. Mitrić, J. Chem. Phys. 115, 10450 (2001).

${ }^{29}$ J. C. Idrobo, S. Ogut, and J. Jellinek, Phys. Rev. B 72, 085445 (2005). 